\title{
Cardiovascular Disease (CVD) Risk Assessment of HIV Medication Regimens using hematopoietic CD34+ progenitor cells
}

\section{Adrian Farid Elzarki}

George Washington University Medical Center

\section{Seshagiri Rao Nandula}

George Washington University Medical Center

Hassan Awal

George Washington University Medical Center

\section{Gary L Simon}

George Washington University Medical Center

Sabyasachi Sen ( $\nabla$ ssen1@gwu.edu )

George Washington University https://orcid.org/0000-0002-3675-9129

\section{Research Article}

Keywords: HIV, CVD, non-nucleoside reverse transcriptase inhibitors, integrase inhibitor

Posted Date: January 12th, 2022

DOI: https://doi.org/10.21203/rs.3.rs-1226890/v1

License: (9) This work is licensed under a Creative Commons Attribution 4.0 International License. Read Full License

Version of Record: A version of this preprint was published at Stem Cell Research \&amp; Therapy on March 7th, 2022. See the published version at https://doi.org/10.1186/s13287-022-02775-6. 


\section{Abstract}

\section{Background}

To determine the effects of integrase inhibitor (INSTI) in comparison to non INSTI based regimens such as non-nucleoside reverse transcriptase inhibitors (NNRTIs) based regimens on cardiovascular disease (CVD) risk in HIV+ patients without overt history of CVD or diabetes, with normal CD4:CD8 count. For CVD risk assessment we primarily used hematopoietic CD34+ progenitor cells, as a biomarker.

\section{Methods}

19 male subjects ages 32-61 years with BMI 21.0- 36.0, were enrolled. This was a single time point, crosssectional, observational study. Subjects were enrolled under 2 groups (either on INSTI based regimen with 13 subjects or NNRTI (non-INSTI) based regimens with 6 subjects) who were taking stable doses of HAART. The medication regimens were a combination of one NRTI (typically tenofovir-emtricitabine) plus one INSTI or NNRTI. Our outcome measures were focused on cardiovascular and endothelial cell function and systemic inflammation. Our primary outcome measures were peripheral blood derived hematopoietic progenitor cell number (CD34 and CD133 positive), CD34+ cell function and gene expression studies. Our secondary outcomes were arterial stiffness measures and serum-based markers of inflammation.

Results

A significant increase in percentage number of progenitor cells, CD133+ cells $(P=0.004)$ was noted along with an increase of double progenitor mark positive CD133+/CD34+ progenitor cell population was observed in INSTI group as compared to NNRTI group, by flow-cytometry. mRNA gene expression for antioxidant gene catalase was noted along with a trend towards a decrease in gene expression of inflammatory marker IL6 $(p=0.06)$ was observed in CD34+ from INSTI group vs NNRTI group. The plasma IL- 6 and CRP levels did not change significantly between the groups. Neutrophil-Lymphocyte ratio (NLR), an important marker of inflammation, was noted to be lower in INSTI group. A mean fasting glucose level was also lower in the INSTI group compared to NNRTI group $(p=0.03)$. Interestingly, UrineMicroalbumin levels were higher in the INSTI group compared to NNRTI group $(p=0.08)$, while eGFR levels were lower in the INSTI group $(p=0.002)$. The arterial stiffness measures did not show statistically significant differences between the two groups.

\section{Conclusion}

We conclude that the INSTI regimen may provide a better CVD risk profile compared to NNRTI based HAART regimen; however the increased albuminuria along with lower eGFR, noted in INSTI group is of concern. Because of the small size, these results would need replication in additional studies before changing clinical practice.

Clinical Trial Registration 
https://clinicaltrials.gov/ct2/show/NCT03782142?

cond=Hiv\&spons=Sabyasachi+sen\&cntry=US\&state=US\%3ADC\&city=Washington\&draw=2\&rank=1

ClinicalTrials.gov Identifier: NCT03782142

\section{Introduction}

Human Immunodeficiency Virus (HIV) is a retrovirus belonging to the lentivirus genus that attacks the human immune system over long periods of time [1]. The prevalence of HIV has been described as a global epidemic [2,3]. According to the CDC, in 2018 nearly 38,000 new cases in the United States and 1.7 million new cases worldwide were reported. The total number of reported individuals with HIV reached approximately 38 million globally. Meanwhile, an estimated 770,000 individuals died as a result of HIVAIDS related illness that same year [3].

Cardiovascular diseases (CVD) are one of the leading causes of morbidity and mortality in antiretroviral treated people living with HIV (PWH) with risk score algorithms based on traditional risk factors being shown to be consistently unreliable in estimating risk in this population. Although persistent associations with inflammatory markers and CVD have been demonstrated, few biomarkers have emerged as being clinically useful. [4]. Cell based assays in the realm of HIV and CVD risk assessment are almost nonexistent although presence of endothelial cell dysfunction in HIV has been well established [5].

There are number of major classes of antiretroviral therapy (ART) which are currently in use. Athough their toxicity profile has been investigated [6], effects on long term CVD risk in HIV remains largely unexplored, The three major categories of medication are as follows: protease inhibitors (PI), integrase strand inhibitors (INSTI), and nucleoside and (NRTIs) and non-nucleoside reverse transcriptase inhibitors (NNRTIs) [7].

Currently, most patients are treated with combination antiretroviral therapy initiated at the time of diagnosis. Combination therapy is utilized to reduce the risk of mutational resistance [8]. Currently, the most frequently employed regimens involve a combination of NRTIs, NNRTIs and/or integrase inhibitors (INSTIS).

HIV infection and some of the antiretroviral (ART) regimens have been associated with adipose tissue/ lipodystrophic changes and disorders of glucose and lipid metabolism. Metabolic and endocrine perturbations including insulin resistance, diabetes, and dyslipidemia have been of significant concern in human immunodeficiency virus (HIV)-infected individuals $[9,10]$.

HIV-infected individuals may be at risk of accelerated atherosclerotic cardiovascular disease (CVD) and metabolic syndrome. More recent data suggest that immune activation and inflammation from chronic HIV infection may also play an important role in HIV-associated metabolic dysfunction [11].

The long-term effects of ART on the cardiovascular system, particularly the newer generation regimens containing INSTI or NNRTI's effect on endothelial cell function and cardiovascular system, have not been 
investigated.

Although the etiology of metabolic/endocrine complications in HIV-infected individuals despite viremic control is poorly understood, it is most likely related to the interplay of host, viral, and ART factors and the complex interactions among the long-term consequences of infection, chronic ART, and the underlying inflammatory process. As noted above it is important to determine which ART regimens promote or reduce the chances of endothelial and cardiometabolic dysregulation, particularly chronically.

We hypothesize that HIV regimens will lead to differences in the degree of cardio-metabolic derangement and stem cell number, function and gene expression. Our goal was to establish or refute the cardiovascular benefits of using an INSTI based HAART regimen using hematopoietic cell (CD34+) based results.

\section{Methods}

In our study we decided to use adult stem cells such as hematopoietic progenitor cells (CD34+) as our primary outcome measure [12]. Our laboratory group has used hematopoietic cells as a cell based diagnostic and prognostic biomarker in multiple scenarios and have evaluated ECD evaluation modalities such as arterial stiffness measures [13], flow mediated dilatation and podocyte inflammatory markers [14-17].

This is a cross-sectional observational study examining patients with HIV and a history of taking either INSTI or NNRTI regimens for at least one year. The study was conducted in accordance with Good Clinical Practice guidelines set forth by the International Conference of Harmonization as well as local regulatory guidelines with the approval and oversight of the George Washington University Institutional Review Board.

Subjects were initially pre-screened to assess eligibility. Once determined preliminary eligibility, they were brought in for a screening visit to confirm eligibility via interview, medical record check and laboratory workup once the subject signed the informed consent form.

The study entailed a single timepoint visit. The assessments that were completed include: vital measurements such as $\mathrm{BMI}$, blood pressure, heart rate, adverse event (AE) checks, and a peripheral blood draw. Approximately $60 \mathrm{ml}$ of blood was drawn for CD34+ endothelial progenitor cell harvesting and routine blood work. Other parameters tested were measurement of waist-to-hip ratio, Tanita body composition scale, pulse wave analysis and pulse wave velocity to determine arterial stiffness.

A follow-up phone call visit was completed 30 days from the last in-person visit to assess for any residual adverse events (AE).

Participants 
The subjects were included if they were males between 40 and 70 years old. Patients with BMI between 25 and $39.9 \mathrm{~kg} / \mathrm{m}^{2}$ were included. We avoided subjects with severe obesity (BMI $\geq 40$ ) as those subjects are deemed to have pre-existing CVD risk). Patients with normal and mildly impaired renal function were included, with lowest eGFR cut-off of $50 \mathrm{ml} / \mathrm{min} / 1.73$ (GFR, as calculated by MDRD formula).

Any patients with uncontrolled hyperglycemia, history of liver disease, clinically significant RBC cell disorders, HBV or HCV, chronic malabsorption, statin medication use, use of consistent steroid medications, untreated thyroid disease was excluded. Additional Inclusion and Exclusion criteria can be found in the Appendix.

Outcome objectives

The objective is to detect differences between INSTI and NNRTI based HAART based regimens Our primary outcome measures were cellular outcome measures pertaining to the cardiovascularhematopoietic system with secondary outcome measures being measures of vascular function such as Arterial Stiffness [measured by pulse wave analysis (Augmentation Index) and pulse wave velocity $(\mathrm{m} / \mathrm{s})$ ], and Serum Biochemistry Analysis pertaining to cardio-metabolic function parameters: such as Hemoglobin $\mathrm{A} 1 \mathrm{C}(\mathrm{HbA} 1 \mathrm{C})$ (as a reflection of glycemic control), Fasting Lipid Profile, C-Reactive Protein (inflammation), Adiponectin (measure of endothelial paracrine function), Fasting Insulin levels (measure of insulin resistance), and Interleukin-6 (inflammation).

The cell-based measures evaluating the function of CD34+ cells were to ascertain the effects of NNRTI and INSTI based regimens on CD34+ cell number, (CD34+ cell number percentage, (\%CD34+ve out of total mononuclear cells, MNC, population), CD34 + cell function (cell migration function in response to a chemotactic agent, stromal derived factor-1a, SDF1a) and CD34+ cell gene expression (specifically genes related to endothelial function) in patients with HIV.

\section{Secondary Outcome Measures}

Body composition measurement

Body composition was measured manually as well as using Tanita ${ }^{\mathrm{TM}}$ BF-350 Body Composition Scale (an impedance scale). Manual measurement included height, waist circumference, hip circumference and BMI calculation. Tanita scale (Tanita Corporation of America, Inc, USA) uses a bio-impedance electrical impulse to measure body fat percent, fat mass $(\mathrm{kg})$, fat-free mass $(\mathrm{kg})$, percent body water, and water mass $(\mathrm{kg})$ alongside weight. It also calculates the subject's BMI and estimated basal metabolic rate (BMR).

\section{Arterial stiffness}

This parameter was measured using Atcor Sphygmocor CP system (Atcor Technologies, USA). We obtained two outcomes: pulse wave velocity and pulse wave analysis. 
Pulse wave analysis (PWA) was measured on the left Radial Artery with the subject supine. At least three readings were taken with Operator Index $\geq 80$. Measurement includes Augmentation Index (AI), Augmentation Index adjusted for Heart Rate of 75 (Al-75), Augmentation Pressure (AP), Aortic and Radial reading of systolic, diastolic, pulse pressure and mean pressure.

Pulse wave velocity (PWV) was measured with the subject supine. This measurement requires a distal and proximal artery point delineation. Right femoral artery was used as the distal point with proximal being the left carotid. A straight line if drawn between these two points would include the heart. Index and ring fingers were used to manually localize the pulse, sometimes an arterial Doppler was used to localize the femoral pulse on patient with challenging body habitus. Once a stable pulse waveform was observed, the probe position was kept stable for 20 more pulses before the reading was finalized. Three readings were taken with standard deviation of less than $10 \%$. The result reported a velocity in $\mathrm{m} / \mathrm{s}$, with standard error of the mean.

Biological sample and vital statistics collection

A venous blood sample was collected from the Antecubital fossa. About $60 \mathrm{~mL}$ of blood was collected, centrifuged at $4^{\circ} \mathrm{C}$, and plasma stored at $-80^{\circ} \mathrm{C}$ until analysis or sent immediately to LabCorp. $60 \mathrm{ml}$ for EPC analysis and $20 \mathrm{ml}$ for standard of care blood tests which included Basic Metabolic Panel, Lipid Panel, HbA1c, fasting glucose, hsCRP, IL6, Adiponectin and Insulin. Urine sample was collected for urine Microalbumin and Creatinine ratio. Vital statistics were measured on the left arm including systolic pressure, diastolic pressure and heart rate, and long sublingual temperature.

\section{Cellular and Clinical Assessments}

\section{CD34+ Endothelial Progenitor Cell Analysis}

Peripheral blood samples (approximately $60 \mathrm{ml}$ ) were drawn from patients and phosphate buffered saline (1:1) was added. Identification and quantification of circulating cell phenotypes was performed on fresh blood samples, within 3 hours after collection, using flow cytometry. Briefly, mononuclear cells (MNCs) were then isolated from whole blood using a Ficoll density centrifuge method. MNCs were counted and an aliquot was used for CFU-Hill colony formation assay following the manufacturer's instruction (Stem Cell Technologies, Vancouver, BC, Canada). Colony forming units were counted at day14. A fraction of the MNC were stained with fluorescein isothiocyanate (FITC)-conjugated antihuman CD34, Allophycocyanin (APC) conjugated antihuman CD184 (CXCR4), Allophycocyanin (APC) conjugated antihuman CD133 and FITC conjugated antihuman CD31 antibodies (Miltenyi Biotec GmbH, BergischGladback, Germany) in order to analyze specific progenitor cell surface markers (CD34 and CD133) and mature endothelial cell surface markers (CD31) or receptors for SDF1a ligand, CXCR4) by flow cytometry. After gating mononuclear cells in the side scatter (SSC)-A vs forward scatter (FSC)-A plot, CD34/CD33/CD184 single- and double-positive cells were identified. Cells were acquired on a fluorescence-activated cell sorter (FACS) Canto instrument (Becton Dickinson, USA) and scored with the Flo-Jo software. 
To isolate EPCs (CD34+), MNCs were magnetically sorted through a column after cells were stained with CD34+ve microbeads antibody (Miltenyi Biotec $\mathrm{GmbH}$, Bergisch Gladback, Germany). An aliquot of CD34+ cells were then stained with trypan blue and counted using an Auto Cellometer Mini (Nexcelom Bioscience, USA) to assess viability.

CD34+ gene expression analysis was performed by quantitative reverse transcriptase polymerase chain reaction (qRT-PCR) as previously described (33). CD34+ cell total mRNA was extracted and purified using the RNeasy Minikit (Qiagen, Germany). mRNA was then converted into cDNA by using the highcapacity cDNA reverse transcriptase kit (Thermo Fisher Scientific, MA). Possible gene expression changes were assessed by a CFX96 real-time PCR systems (Bio-Rad, CA.) using Taqman Universal masters Mix II (Thermo Fisher Scientific, USA) and inventoried probes. The gene expression analysis included antioxidants, apoptosis, endothelial functions, chemotaxis, inflammation, and endothelial lineage cell surface markers. The expression of each individual gene was normalized to either housekeeping $18 \mathrm{~S}$ and mean $\mathrm{Cq}$ values are reported.

The migratory capacity of CD34+ was evaluated using the CytoSelect 24-well Cell Migration Assay kit (Cell Biolads, Inc., San Diego, CA). Cells were suspended in Serum free media and seeded at 100,000 cells per insert. Migration of the cells through a 3um polycarbonate membrane to the wells containing a serum-free media (control) and chemoattractant SDF-1a (10 or $100 \mathrm{ng} / \mathrm{mL}$ ) (from Sigma-Aldrich, USA) was assessed after cells were kept overnight in incubator. Migratory cells were dissociated from the membrane and subsequently lysed and quantified by fluorescence $(480 \mathrm{~nm} / 530 \mathrm{~nm})$ using CyQuant GR dye (Cells Biolabs, Inc, USA). The fluorescence ratios between cells exposed to the chemotactic factor and cells exposed to chemoattractant-free media (control) along the visits were used to analyze the migratory capacity of the cells.

\section{Statistical Methods}

Because of the small sample size in this pilot program study, the analysis emphasis is on descriptive statistics. Differences between the INSTI and NNRTI groups were compared by Mann-Whitney tests. Significance test results indicate rough markers of parameters which may be affected by treatment regimen and not as confirmation of treatment effects. To balance Type 1 and 2 error rates for this preliminary study, no adjustments were made for multiple testing.

\section{Results}

Study Sample. A total of 19 subjects were enrolled into two different groups taking two different classes of Antiretroviral regimen, either INSTI based regimen (13 subjects) or NNRTI based regimen (6 subjects). After several months of recruitment attempts, it was evident that recruiting more than a handful of patients in the Protease Inhibitors group would not be successful. We therefore dropped that group. We were able to attain a final sample size of $n=13$ for the INSTI group and $n=6$ patients in the NNRTI group. 
Characteristics of the 2 groups of patients are shown in Table 1. Outcome differences are in Table 2.

Table 3 shows the prominent components of the HAART regimen for each subject included in either of the two groups.

\section{Cellular Outcomes:}

\section{Endothelial progenitor cell (CD34+) based assays}

Total number of MNCs ( $1.10 \times 10^{8}$ for INSTI group and $1.01 \times 10^{8}$ for NNRTI group) and the \% of endothelial progenitor cells (CD34t) purified from MNCs population were slightly higher in INSTI group (1.57 \pm 0.05$)$ as compared to NNRTI group (1.33 \pm 0.54$)$, although not statistically significant.

Colony Forming Units (CFU Assay): Results suggest higher CFU values in the NNRTI group (11.73 \pm 3.86 in INSTI group and $16.37 \pm 3.10$ in NNRTI group) but not statistically significant, $p=0.16$ ).

The migratory response of CD34+ cells to the chemotactic factor SDF1a (concentration of $10 \mathrm{ng} / \mathrm{ml}$ ), an important functional property and assay of progenitor cells, was higher in INSTI group $(0.83 \pm 0.13)$ as compared to NNRTI group (1.21 \pm 0.25$)$, even though statistically not significant. Lesser distance from chemotactic factor target indicates better cell migratory property.

CD133+ progenitor cells (Fig 1A) had a higher level the in INSTI group ( $p=0.004)$ as compared to NNRTI group. CD34+CD133 (dual progenitor cell marker positive, Fig1B) cell number were also higher in the INSTI group with values approaching statistical significance $(p=0.07)$. CD34+ progenitor cell number (single progenitor cell marker positive) were also higher in the INSTI group.

\section{Gene Expression Analysis}

CD34+cell function represents vascular endothelial health, so we investigated the gene expression on CD34+ cells for antioxidants such as cytosolic Catalase, mitochondrial SOD2 (superoxide dismutase 2) and extra-cellular antioxidant GPX3 (glutathione peroxidase 3), of which catalase is the most investigated and an important one. The mean $\mathrm{Cq}$ values of cytoplasmic antioxidant, Catalase was slightly higher $(p=0.13)$ in the INSTI group (Fig. 2A) whereas, a trend in increased mean Cq values for SOD2 $(p=0.57)$ was also observed in INSTI group compared to NNRTI group. The endothelial marker gene expressions, PECAM1 $(p=0.81)$ and VEGF-A $(p=0.47)$ Cq were nearly at the same level for the 2 groups. However, there was a decreased mean Cq value of the inflammatory marker II-6 $(p=0.06)$ noted in the INSTI group (Fig.2B), and no difference is seen in TNF- $a$ value $(p=0.47)$ in INSTI group as compared to NNRTI group. Therefore, taking all hematopoietic stem cell based results together it appears INSTI does provide some degree of vascular endothelial protection compared to NNRTI based HAART regimens.

\section{Clinical Outcomes:}

Table 2 shows blood biochemistry measures collected during the single time visit across the entire study group. Most of the parameters were not statistically significant between groups but were showing a trend 
towards lower systolic and diastolic pressure, BMI and higher Insulin levels in NNRTI group.

\section{Venous Blood Biochemistries}

Venous blood biochemistries were analyzed by LabCorp of America. We found no statistically significant differences in Adipokines such as leptin and adiponectin levels in NNRTI group as compared to INSTI group. Please note Adiponectin particularly has been associated with improved vascular function (See Table. 2, Results).

Serum markers of Insulin Sensitivity: Insulin levels demonstrate no evidence of a statistical difference $(p=0.95)$ between the two arms. In the NNRTI arm the insulin level was $18.86(S E M+10.08)$ while the INSTI arm was 11.66 (SEM+2.14). Although there is no evidence of differences in insulin levels, it is interesting that the mean fasting glucose levels in the NNRTI group are significantly increased $(p=0.03)$ as compared to INSTI group. (Fig. 4).

Inflammatory Markers: We analyzed lipid profile and inflammatory markers such as IL6 and CRP. Interestingly, IL6 showed a mean value in NNRTI arm of 1.72 (SEM+ 0.45) and in the INSTI level arm was 3.04 (SEM+ 0.57), although not statistically significant. CRP, also known as highly sensitive Creactive protein, is an inflammatory marker which is compared between the two groups (Fig. 5B). In NNRTI group the mean value was higher at 2.27(SEM+ 0.69) and in the INSTI group, there was a mean of 1.73 (SEM+ 0.37), statistically similar to each other.

Urine Microalbumin: There was a non-significant trend for lower albuminuria levels in the NNRTI group $(10.75$ SEM+ 4.45) compared to the INSTI group (102.92 SEM+68.05, Fig. 5). For reference, normal albumin levels should be $30 \mathrm{mg} / \mathrm{g}$ or below, with elevated levels implying worsening kidney function. Though non significant the $p$ value was close to significance at 0.08

eGFR: EGFR levels (as shown in Fig. 6) demonstrate a statistically significant ( $p=.002)$ difference in glomerular filtration rate between the INSTI group (59 SEM + 8.04) and NNRTI group (106.16 + 4.94). The data suggests that in the INSTI group there is a suggestion of early stage chronic kidney disease (CKD).

Arterial Stiffness: Stiffness of an artery significantly contributes to lack of pliability and contractility and is an important marker of increased peripheral resistance, diastolic dysfunction and systemic hypertension. The results of arterial stiffness measurements may indicate early stages of kidney damage. It is associated with cardiovascular diseases in older individuals and is positively associated with hypertension, coronary artery disease, stroke, heart failure and atrial fibrillation. Arterial stiffness is assessed using parameters such as Al adjusted for a heart rate of 75 (Al-75) and PWV.

Average Augmentation Index-75 is higher in the INSTI group (17.08 \pm 3.97$)$ as compared to NNRTI group (12.83 \pm 4.85$)$ but not statistically significant. Similar trend was observed with Average Augmentation Pressure with higher values in INSTI group $(9.08 \pm 1.85)$ as compared to NNRTI group $(7.83 \pm 2.85)$. 


\section{Discussion}

This is a novel study where we compared two commonly used HIV medication regimens. Currently there are seven different classes of HIV medications, non-nucleoside reverse transcriptase inhibitors (NNRTIs), Nucleoside reverse transcriptase inhibitors (NRTIs), Protease inhibitors (PIs), Fusion inhibitors, CCR5 antagonists, Integrase strand transfer inhibitors (INSTIs), Post-attachment inhibitors, as eluded before.

An initial antiretroviral regimen generally consists of two nucleoside/nucleotide reverse transcriptase inhibitors (NRTIs) in combination with a third active drug from one of the following classes: nonnucleoside reverse transcriptase inhibitor (NNRTI), protease inhibitor (PI; boosted with ritonavir or cobicistat), or integrase strand transfer inhibitor (INSTI). PI based regimens have been implicated in causing weight gain, dyslipidemia and worsening of insulin resistance [10]. Therefore, we embarked on trying to differentiate between NNRTI and INSTI as part of a medication regimen which contained two NRTI at a baseline, frequently tenofovir-emtricitabine.

As far as the differences between the regimens, the interesting measures were mostly cell based with number of progenitor cells both CD133 and CD34 higher in INSTI group indicating better CVD risk profile, based on published literature [12]. The effects of INSTI versus NNRTIs are consistent with the results from gene expression studies (Figure 2) in which two antioxidants, SOD2 and Catalase mRNA expressions showing higher expressions in INSTI group in CD34+ cells with Catalase result showing statistical difference.

A bonafide cellular marker of inflammation such as Neutrophil-Lymphocyte Ratio (NLR) was also lower in INSTI group (Fig3).

As initial manifestations of metabolic dysfunction, INSTI group showed lower fasting glucose levels versus NNRTI (Figure 4) indicating greater metabolic dysfunction in NNRTI group. Inflammatory marker levels such as interleukin 6 or IL- 6 and hs-CRP were very similar to each other

Itis well known that increased albuminuria is an early sign of cardiovascular abnormality. In both diabetes and metabolic syndrome, one notes the presence of albuminuria ahead of overt expression of other vascular complications [18].

Though the difference failed to reach statistical significance $(p=0.08)$ the mean Urine-Microalbumin was modestly higher in INSTI group compared to NNRTI group (Fig 5). A lower GFR in INSTI group (Fig 6) in the context of high levels of proteinuria is interesting. Higher levels of proteinuria indicates renal podocyte damage. Podocyte damage may be associated with impaired renal function, which is often associated with low eGFR. This maybe a possibility with INSTI treatment.Arterial stiffness parameters [13] did not show statistical difference between the groups.

Considering the results of these analyses, the statistically significant differences such as progenitor cell number increase, increased gene expression of antioxidants and inflammatory markers genes in INSTI group along with NLR values, we conclude that INSTI medication group most likely provides better and 
sustained cardiometabolic risk reduction to HIV subjects. The lower fasting glucose levels were also reassuring in INSTI group.

While our non-cellular results (both plasma values and microalbuminuria) did not show a statistically significant difference, our cellular results, based on CD34+ cells actually did show statistically significant differences in number and protective antioxidant gene expressions such as Catalase and SOD2. Our results once again demonstrate that cellular results may be of more value even if the cohort size is small, compared to non-cellular results, though the latter is more commonly used clinically.

However certain well established clinical parameters such as higher microalbinuria, with poor eGFR along indicates some concern with INSTI group, impacting renal function.

\section{Limitations:}

It is to be acknowledged that this study was designed as preliminary study and results need to be interpreted in that context. The small sample size in the NNRTI group and consequent low statistical power, statistics for the 2 groups needs to be interpreted descriptively (Table 2) though we have calculated the p-values. The most noteworthy differences were clearly cell based assays, however, those differences may be due to chance and will need to be replicated in a larger study.

\section{Conclusions}

We conclude that the INSTI based HAART regimen may provide a better CVD risk profile compared to NNRTI based HAART regimen, based on hematopoietic stem cell based assays, and provides with statistical significant differences between the groups even in this small cohort. Certain clinical parameters however is of concern such as the increased albuminuria along with lower eGFR, noted in INSTI group. Because of the small size, these results would need replication in additional studies.

\section{Abbreviations}

- INSTI - Integrase inhibitor

- NNRTI - Non-nucleotide reverse transcriptase inhibitors

- Al - Augmentation Index

- Al-75 - Augmentation Index adjusted for a heart rate of 75

- AP - Augmentation Pressure

- AS - Arterial stiffness

- BCl-2 - B-cell lymphoma 2

- BMI - Body mass index

- BUN - Blood urea nitrogen

- CAT -- Catalase 
- CDKN1A - cyclin-dependent kinase inhibitor $1 \mathrm{~A}$

- CD34 - Progenitor marker

- CFU - Colony forming units

- CVD - Cardiovascular disease

- CXCR4 - C-X-C Motif Chemokine Receptor 4

- DBP - Diastolic blood pressure

- DPP-4 Inhibitors - Dipeptidyl peptidase-4 inhibitors

- EDN-1 - Endothelin 1

- eGFR - Estimated glomerular filtration rate

- ELISA - Enzyme-Linked Immunosorbent Assay

- eNOS - Nitric Oxide Synthase 3 (Endothelial Cell)

- EPCs - Endothelial Progenitor Cells

- FFM - Fat free mass

- GAPDH - Glyceraldehyde-3-Phosphate Dehydrogenase

- GLP1 - Glucagon Like Peptide 1

- GPX1 - Glutathione Peroxidase 1

- HbA1C - Hemoglobin A1C, glycated hemoglobin test

- IGF1 - Insulin-like growth factor 1

- IL-6 - Interleukin 6

- $\mathrm{kg}$ - Kilograms (weight)

- Ibs - Pounds (weight)

- MNCs - Mononuclear cells

- PECAM1 - Platelet And Endothelial Cell Adhesion Molecule 1

- PWA - Pulse Wave Analysis

- PWV - Pulse Wave Velocity

- qRT-PCR - Quantitative reverse transcriptase polymerase chain reaction

- REE - resting energy expenditure

- SBP - Systolic blood pressure

- SDF1a-Stromal cell-derived factor-1a

- SOD1, SOD2 - Super oxide dismutase $1 \& 2$

- TBW - Total body water

- TNFa - Tumor Necrosis Factor a

- TP53 - Tumor protein p53

- VEGF - vascular endothelial growth factor 
- VEGFA - Vascular Endothelial Growth Factor A

- VEGFR2 - Vascular endothelial growth factor receptor 2

\section{Declarations}

Ethics approval and consent to participate: All subjects enrolled in the trial signed informed consent Consent for publication: Consent for publication was obtained from all participants of the study, coauthors and persons acknowledged

Availability of data and material: All data pertaining to the study are freely available

Competing interests: there are no competing interests

Funding: This publication resulted from research funds given to $\mathrm{Dr}$ Sen by the District of Columbia Center for AIDS Research, an NIH funded program (P30Al117970), which is supported by the following NIH CoFunding and Participating Institutes and Centers: NIAID, NCl, NICHD, NHLBI, NIDA, NIMH, NIA, NIDDK, NIMHD, NIDCR, NINR, FIC and OAR. The content is solely the responsibility of the authors and does not necessarily represent the official views of the $\mathrm{NIH}^{.}$

Authors' contributions: Both AE and SRN contributed equally to sample collection, data analysis, interpretation and manuscript composition. HA: helped in subject enrollment and data compilation, GLS: helped in design of the study, data interpretation and manuscript review, SS: received the grant, designed the study, contributed towards data compilation, data analysis \& interpretation and manuscript compilation. All authors have read and approved the manuscript.

Acknowledgements: We would like to acknowledge help of Sam Simmens, PhD from GW-DC-CFAR Biostatistics Core for providing advice and support on statistical analysis.

\section{References}

1. Rajesh Thippeshappa, Patricia Polacino, Shaswath S. Chandrasekar, Khanghy Truong, Anisha Misra, Paula C. Aulicino, Shiu-Lok Hu, Deepak Kaushal, and Jason T. Kimata . In vivo Serial Passaging of Human-Simian Immunodeficiency Virus Clones Identifies Characteristics for Persistent Viral Replication. Front Microbiol. 2021; 12: 779460.

2. Ward Z, Stone J, Bishop C, Ivakin V, Eritsyan K, Deryabina A, Low A, Cepeda J, Kelly SL, Heimer R, Cook R, Altice FL, Litz T, Terlikbayeva A, El-Bassel N, Havarkov D, Fisenka A, Boshnakova A, Klepikov A, Saliuk T, Deshko T, Vickerman P. Costs and impact on HIV transmission of a switch from a criminalisation to a public health approach to injecting drug use in eastern Europe and central Asia: a modelling analysis. Lancet HIV. 2021 Dec 9: S2352-3018(21)00274-5. doi: 10.1016/S23523018(21)00274-5. 
3. Centers for disease control and prevention. Diagnoses of HIV infection, by race/ethnicity and selected characteristics, 2019. HIV Surveillance Supplemental Report. 2019;32;1-123.

4. McGettrick P, Mallon PWG. Biomarkers to predict cardiovascular disease in people living with HIV. Curr Opin Infect Dis. 2021 Dec 3. doi: 10.1097/QC0.0000000000000802. Epub ahead of print. PMID: 34873078.

5. Mezoh G, Crowther NJ. Deciphering Endothelial Dysfunction in the HIV-Infected Population. Adv Exp Med Biol. 2019; 1134:193-215. doi: 10.1007/978-3-030-12668-1_11. PMID: 30919339.

6. Vos AG, Venter WDF. Cardiovascular toxicity of contemporary antiretroviral therapy. Curr Opin HIV AIDS. 2021 Nov 1;16(6):286-291. doi: 10.1097/COH.0000000000000702. PMID: 34545036.

7. Cunha RF, Simões S, Carvalheiro M, Pereira JMA, Costa Q, Ascenso A. Novel Antiretroviral Therapeutic Strategies for HIV. Molecules. 2021 Aug 31;26(17):5305. doi: 10.3390/molecules26175305. PMID: 34500737; PMCID: PMC8434305.

8. Blassel L, Tostevin A, Villabona-Arenas CJ, Peeters M, Hué S, Gascuel O; UK HIV Drug Resistance Database. Using machine learning and big data to explore the drug resistance landscape in HIV. PLoS Comput Biol. 2021 Aug 26;17(8): e1008873. doi: 10.1371/journal.pcbi.1008873. PMID: 34437532; PMCID: PMC8425536.

9. Hsue PY, Waters DD. HIV infection and coronary heart disease: mechanisms and management. Nat Rev Cardiol. 2019 Dec;16(12):745-759. doi: 10.1038/s41569-019-0219-9. Epub 2019 Jun 10. PMID: 31182833.

10. Feinstein MJ, Hsue PY, Benjamin LA, Bloomfield GS, Currier JS, Freiberg MS, Grinspoon SK, Levin J, Longenecker CT, Post WS. Characteristics, Prevention, and Management of Cardiovascular Disease in People Living With HIV: A Scientific Statement from the American Heart Association. Circulation. 2019 Jul 9;140(2): e98-e124.

11. Vos AG, Chersich MF, Klipstein-Grobusch K, Zuithoff P, Moorhouse MA, Lalla-Edward ST, Kambugu A, Kumarasamy N, Grobbee DE, Barth RE, Venter WD. Lipid levels, insulin resistance and cardiovascular risk over 96 weeks of antiretroviral therapy: a randomised controlled trial comparing low-dose stavudine and tenofovir. Retrovirology. 2018 Dec 14;15(1):77. doi: 10.1186/s12977-018-0460-z. PMID: 30547820; PMCID: PMC6295103.

12. Jain $\mathrm{R}$, Awal $\mathrm{H}$, Sen $\mathrm{S}$. Using adult stem cells to monitor endothelial dysfunction in diabetes mellitus. J Diabetes Complications. 2020 Jul;34(7):107588. doi: 10.1016/j.jdiacomp.2020.107588. Epub 2020 Apr 19. PMID: 32345465.

13. Khiyami AM, Dore FJ, Mammadova A, Amdur RL, Sen S. The Correlation of Arterial Stiffness with Biophysical Parameters and Blood Biochemistry. Metab Syndr Relat Disord. 2017 May;15(4):178182. doi: 10.1089/met.2016.0136. Epub 2017 Feb 22. PMID: 28437199.

14. Nandula SR, Kundu N, Awal HB, Brichacek B, Fakhri M, Aimalla N, Elzarki A, Amdur RL, Sen S. Role of Canagliflozin on function of CD34+ve endothelial progenitor cells (EPC) in patients with type 2 diabetes. Cardiovasc Diabetol. 2021 Feb 13;20(1):44. doi: 10.1186/s12933-021-01235-4. PMID: 33581737; PMCID: PMC7881606. 
15. Awal HB, Nandula SR, Domingues CC, Dore FJ, Kundu N, Brichacek B, Fakhri M, Elzarki A, Ahmadi N, Safai S, Fosso M, Amdur RL, Sen S. Linagliptin, when compared to placebo, improves CD34+ve endothelial progenitor cells in type 2 diabetes subjects with chronic kidney disease taking metformin and/or insulin: a randomized controlled trial. Cardiovasc Diabetol. 2020 Jun 3;19(1):72. doi: 10.1186/s12933-020-01046-z. PMID: 32493344; PMCID: PMC7271387.

16. Dore FJ, Domingues CC, Ahmadi N, Kundu N, Kropotova Y, Houston S, Rouphael C, Mammadova A, Witkin L, Khiyami A, Amdur RL, Sen S. The synergistic effects of saxagliptin and metformin on CD34+ endothelial progenitor cells in early type 2 diabetes patients: a randomized clinical trial. Cardiovasc Diabetol. 2018 May 3;17(1):65. doi: 10.1186/s12933-018-0709-9. PMID: 29724198; PMCID: PMC5934787.

17. Levy ME, Anastos K, Levine SR, Plankey M, Castel AD, Molock S, Sen S, Asch FM, Milam J, Aouizerat B, Weber KM, Golub ET, Kaplan RC, Kassaye S. Depression and Psychosocial Stress Are Associated With Subclinical Carotid Atherosclerosis Among Women Living With HIV. J Am Heart Assoc. 2020 Jul 7;9(13): e016425. doi: 10.1161/JAHA.120.016425. Epub 2020 Jun 22. PMID: 32564652; PMCID: PMC7670495.

18. Lagathu C, Béréziat V, Gorwood J, Fellahi S, Bastard JP, Vigouroux C, Boccara F, Capeau J. Metabolic complications affecting adipose tissue, lipid and glucose metabolism associated with HIV antiretroviral treatment. Expert Opin Drug Saf. 2019 Sep;18(9):829-840. doi:

10.1080/14740338.2019.1644317.

\section{Tables}

Table. 1: Baseline Characteristics between the two groups. 


\begin{tabular}{|llll|}
\hline Parameter & INSTI $(\mathrm{n}=13)$ & NNRTI $(\mathrm{n}=6)$ & Mann-Whitney P-value \\
\hline Age & $49.46(33-61)$ & $44.5(32-59)$ & \\
\hline Sex (male only) & 13 & 6 & \\
\hline African American & 11 & 3 & \\
\hline Caucasian & 2 & 3 & \\
\hline & & & \\
\hline Systolic & $135.30 \pm 7.53$ & $132.33 \pm 8.17$ & 0.9488 \\
\hline Diastolic & $83.76 \pm 3.76$ & $80 \pm 4.47$ & 0.8483 \\
\hline Pulse & $74.46 \pm 3.51$ & $68.66 \pm 6.07$ & 0.2916 \\
\hline Temp & $97.98 \pm 0.13$ & $98.48 \pm 0.11$ & 0.0283 \\
\hline BMI & $27.42 \pm 1.28$ & $26.74 \pm 1.99$ & 0.8025 \\
\hline Weigh Kgs & $82.04 \pm 3.88$ & $76.36 \pm 9.80$ & 0.7166 \\
\hline Waist in CM & $95.08 \pm 3.67$ & $93.4 \pm 4.84$ & 0.8995 \\
\hline Hip in CM & $103.25 \pm 2.98$ & $102.6 \pm 3.98$ & 0.9360 \\
\hline
\end{tabular}

Table. 2: All Results between two groups. Results that are significant or close to significance (at or below $p=0.05$ ), are marked in Red. Results that are greater than $p=0.05$ but less then $p=0.2$ are marked in green. 


\begin{tabular}{|c|c|c|c|}
\hline Parameters & INSTI (n=13) & NNRTI (n=6) & $\begin{array}{l}\text { Mann-Whitney P- } \\
\text { value }\end{array}$ \\
\hline Avg PWV & $8.72 \pm 0.50$ & $7.62 \pm 0.78$ & 0.1998 \\
\hline Avg Aug Index_75 & $17.08 \pm 3.97$ & $12.83 \pm 4.85$ & 0.3974 \\
\hline Avg Aug Index & $21 \pm 3.86$ & $18.16 \pm 3.96$ & 0.2587 \\
\hline Avg Aug Pressure & $9.08 \pm 1.85$ & $7.83 \pm 2.85$ & 0.4202 \\
\hline Glucose & $91.58 \pm 3.71$ & $100.66 \pm 2.78$ & 0.0379 \\
\hline Uric Acid & $5.75 \pm 0.35$ & $5.61 \pm 0.76$ & 0.8379 \\
\hline BUN & $15.45 \pm 1.60$ & $13.66 \pm 1.68$ & 0.6454 \\
\hline Creatinine (Serum) & $1.15 \pm 0.05$ & $0.92 \pm 0.05$ & 0.2838 \\
\hline Sodium & $140.33 \pm 0.89$ & $140.33 \pm 0.91$ & 0.8378 \\
\hline Potassium & $4.30 \pm 0.12$ & $4.2 \pm 0.11$ & 0.9149 \\
\hline Chloride & $103 \pm 0.93$ & $104 \pm 0.68$ & 0.7990 \\
\hline AST (SGOT) & $41.25 \pm 16.53$ & $28 \pm 6.63$ & 0.8685 \\
\hline ALT (SGPT) & $43 \pm 20.14$ & $30 \pm 7.18$ & 0.4227 \\
\hline Cholesterol & $178.16 \pm 14.65$ & $203.66 \pm 11.55$ & 0.4240 \\
\hline Triglycerides & $139 \pm 28.99$ & $128.83 \pm 19.97$ & 0.6317 \\
\hline HDL Cholesterol & $53.25 \pm 4.18$ & $53 \pm 6.02$ & 0.8729 \\
\hline VLDL Cholesterol & $27 \pm 5.70$ & $24 \pm 3.44$ & 0.6655 \\
\hline LDL Cholesterol & $97.91 \pm 11.25$ & $126.66 \pm 12.52$ & 0.1306 \\
\hline LDL/HDL & $1.94 \pm 0.24$ & $2.6 \pm 0.46$ & 0.2496 \\
\hline URINE- Creatinine & $132.76 \pm 18.78$ & $139.3 \pm 35.69$ & 0.99 \\
\hline URINE- Microalbumin & $102.92 \pm 68.05$ & $10.75 \pm 4.45$ & 0.0851 \\
\hline $\begin{array}{l}\text { URINE- Microalb/Creat } \\
\text { Ratio }\end{array}$ & $66.76 \pm 36.71$ & $8.46 \pm 2.53$ & 0.2091 \\
\hline eGFR & $59 \pm 8.04$ & $106.16 \pm 4.94$ & 0.0022 \\
\hline Serum CRP & $1.73 \pm 0.37$ & $2.27 \pm 0.69$ & 0.4936 \\
\hline Serum IL6 & $3.04 \pm 0.57$ & $1.72 \pm 0.45$ & 0.6250 \\
\hline Total WBC & $5.54 \pm 0.44$ & $5.66 \pm 1.29$ & 0.6657 \\
\hline
\end{tabular}




\begin{tabular}{|c|c|c|c|}
\hline Total RBC & $4.50 \pm 0.13$ & $4.95 \pm 0.30$ & 0.2496 \\
\hline Hemoglobin & $13.59 \pm 0.45$ & $14.63 \pm 0.35$ & 0.1856 \\
\hline Hematocrit & $40.90 \pm 1.08$ & $42.81 \pm 1.36$ & 0.4792 \\
\hline MCV & $90 \pm 1.41$ & $86.83 \pm 2.61$ & 0.2251 \\
\hline $\mathrm{MCH}$ & $30.26 \pm 0.83$ & $29.95 \pm 1.34$ & 0.8375 \\
\hline $\mathrm{MCHC}$ & $33.15 \pm 0.48$ & $34.36 \pm 0.60$ & 0.0973 \\
\hline RDW & $13.91 \pm 0.24$ & $13.41 \pm 0.72$ & 0.2793 \\
\hline Platelet & $230 \pm 15.87$ & $204.83 \pm 22.58$ & 0.2500 \\
\hline Neutrophil & $46.66 \pm 3.82$ & $58 \pm 2.19$ & 0.0870 \\
\hline Lymphocyte & $40.25 \pm 3.12$ & $32 \pm 1.89$ & 0.1299 \\
\hline Monocyte & $9.16 \pm 0.66$ & $7.66 \pm 0.55$ & 0.1648 \\
\hline HbA1c & $5.32 \pm 0.13$ & $5.18 \pm 0.20$ & 0.6016 \\
\hline Leptin & $13.51 \pm 3.69$ & $8.43 \pm 2.33$ & 0.6165 \\
\hline Adiponectin & $4.58 \pm 0.81$ & $5.06 \pm 1.26$ & 0.8262 \\
\hline Insulin & $11.66 \pm 2.14$ & $18.86 \pm 10.08$ & 0.9501 \\
\hline MNC & $\begin{array}{l}1.10 \mathrm{E}+08 \pm \\
1.44 \mathrm{E}+07\end{array}$ & $\begin{array}{l}1.01 \mathrm{E}+08 \pm \\
1.89 \mathrm{E}+07\end{array}$ & 0.7815 \\
\hline \%CD34+ & $1.57 \pm 0.05$ & $1.33 \pm 0.54$ & 0.9157 \\
\hline SDF10/control & $0.83 \pm 0.13$ & $1.21 \pm 0.25$ & 0.2786 \\
\hline CFU Day 14 & $11.73 \pm 3.86$ & $16.37 \pm 3.10$ & 0.1697 \\
\hline \multicolumn{4}{|l|}{$\begin{array}{l}\text { CD34+cell Gene } \\
\text { Expressions }\end{array}$} \\
\hline CAT & $33.50 \pm 1.13$ & $30.48 \pm 0.64$ & 0.1320 \\
\hline SOD2 & $33.12 \pm 1.62$ & $29.17 \pm 0.71$ & 0.5728 \\
\hline VEGFA & $34.50 \pm 0.95$ & $36.29 \pm 0.78$ & 0.4762 \\
\hline PECAM1 & $34.43 \pm 1.42$ & $32.01 \pm 0.68$ & 0.8182 \\
\hline EDN1 & $36.01 \pm 0.18$ & $37.1 \pm 0.62$ & 0.2333 \\
\hline
\end{tabular}




\begin{tabular}{|llll|}
\hline NOS3 & $39.4 \pm 0.27$ & $39.11 \pm 0.73$ & 0.7619 \\
\hline TPKN3 & $32.56 \pm 1.22$ & $32.39 \pm 0.82$ & 0.7922 \\
\hline IL6 & $33.37 \pm 0.89$ & $31.68 \pm 0.76$ & 0.2662 \\
\hline TNF & $36.65 \pm 0.63$ & $38.81 \pm 0.36$ & 0.0635 \\
\hline GPX3 & $34.10 \pm 0.60$ & $35.32 \pm 0.82$ & 0.4762 \\
\hline CXCL12 & $38.82 \pm 1.03$ & $38.27 \pm 0.88$ & 0.9307 \\
\hline CXCR4 & $38.97 \pm 0.26$ & $37.71 \pm 0.30$ & 0.2286 \\
\hline Flow Cytometry & $33.56 \pm 1.41$ & $31.89 \pm 0.86$ & 0.6991 \\
\hline \% of CD34-FITC & & & \\
\hline \% of CD184-APC & $4.67 \pm 143$ & $3.68 \pm 1.06$ & 0.9451 \\
\hline \% of CD31-FITC & $74.6 \pm 2.77$ & $55.9 \pm 9.25$ & 0.1059 \\
\hline \% of CD133 APC & $84.23 \pm 8.17$ & $43.84 \pm 15.70$ & 0.0539 \\
\hline \% of CD34FITC+CD184+ & $8.83 \pm 2.23$ & $0.25 \pm 0.15$ & 0.0040 \\
\hline APC & $11.78 \pm 5.59$ & $2.95 \pm 0.69$ & 0.5395 \\
\hline \% of & & & 0.3736 \\
\hline CD31FITC+CD184APC+ & $63.13 \pm 6.89$ & $40.30 \pm 11.50$ & 0.0759 \\
\hline $\begin{array}{l}\text { \% of CD34 FITC+CD133 } \\
\text { APC }\end{array}$ & $2.0 \pm 0.96$ & $0.29 \pm 0.15$ & \\
\hline
\end{tabular}

Table 3: Shows medications and doses of all 21 subjects enrolled in the study 


\begin{tabular}{|c|c|c|c|c|}
\hline Patient & Medication Group & Medication Name & Dose/Unit & Freq \\
\hline 1 & INSTI & Descovy +lsentress & 600 (2)-200-25 & QD \\
\hline 2 & INSTI & Truvada \&Tivicay & 200 & QD \\
\hline 3 & INSTI & Triumeq & $600-50-300$ & QD \\
\hline 4 & INSTI & Triumeq & $600-50-300$ & QD \\
\hline 5 & INSTI & Genvoya & $150-150-200$ & QD \\
\hline 6 & INSTI & Biktarvy & $50-200-25$ & QD \\
\hline 7 & INSTI & Tivicay \& Descovy & $50-200-25$ & QD \\
\hline \multicolumn{5}{|l|}{8} \\
\hline 9 & INSTI & Juluca & $50-25$ & QD \\
\hline 10 & NNRTI & Odefsey (Rilpivirine) & $200-25-25$ & QD \\
\hline 11 & INSTI & Juluca & $50-25$ & QD \\
\hline 12 & INSTI & Genvoya & $150-150-200$ & QD \\
\hline 13 & NNRTI & Odefsey & $200-25-25$ & QD \\
\hline 14 & NNRTI & Atripla (Efivarenz) & $600-200-300$ & QD \\
\hline 15 & & Patient Dropped Out & & \\
\hline 16 & NNRTI & Odefsey & $200-25-25$ & QD \\
\hline 17 & INSTI & Biktarvy & $50-200-25$ & QD \\
\hline 18 & NNRTI & Odefsey & $200-25-25$ & QD \\
\hline 19 & NNRTI & Epzicom+Viramune & $600-300$ & QD \\
\hline \multicolumn{5}{|l|}{20} \\
\hline 21 & INSTI & Descovy+Isentress & $200-25$ & QD \\
\hline 22 & INSTI. & Descovy+Tivacay & $200-25$ & QD \\
\hline
\end{tabular}

\section{Figures}



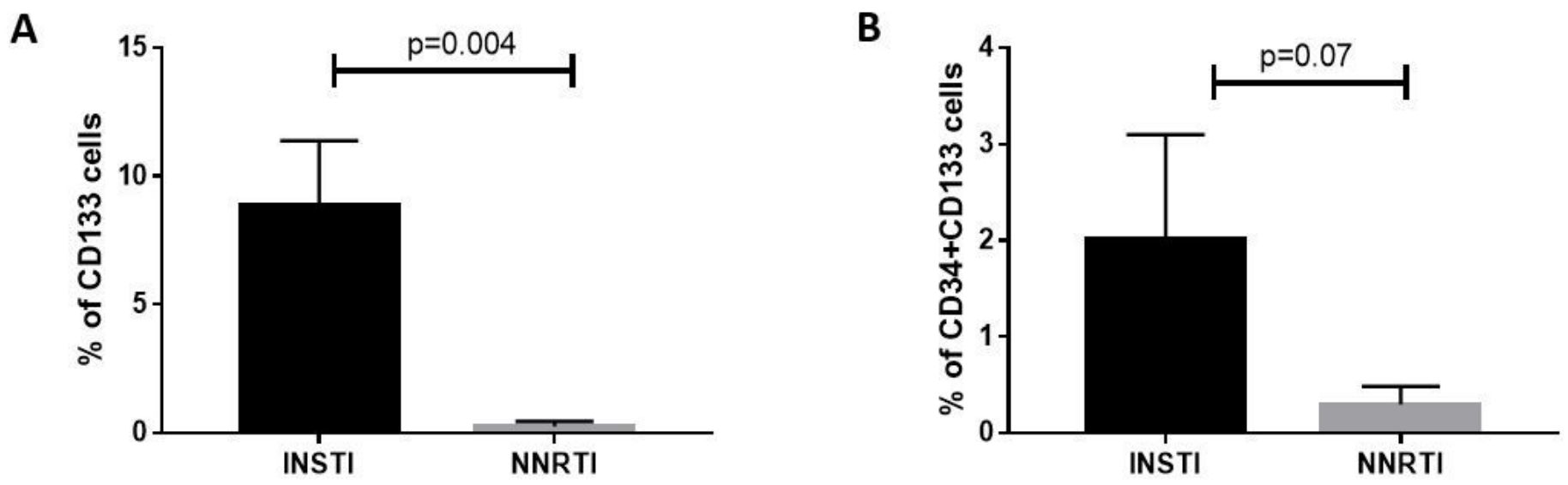

\section{Figure 1}

Cell Based Changes: A significant increase in percentage of CD133 positive hematopoietic cells (Fig.1A, $\mathrm{p}=0.004)$

A trend in increased dual positive CD34+ and CD133+ cells, close to statistical significance (Fig.1B; $\mathrm{p}=0.07)$ is observed in INSTI $(n=13)$ group as compared to NNRTI $(n=6)$ group.
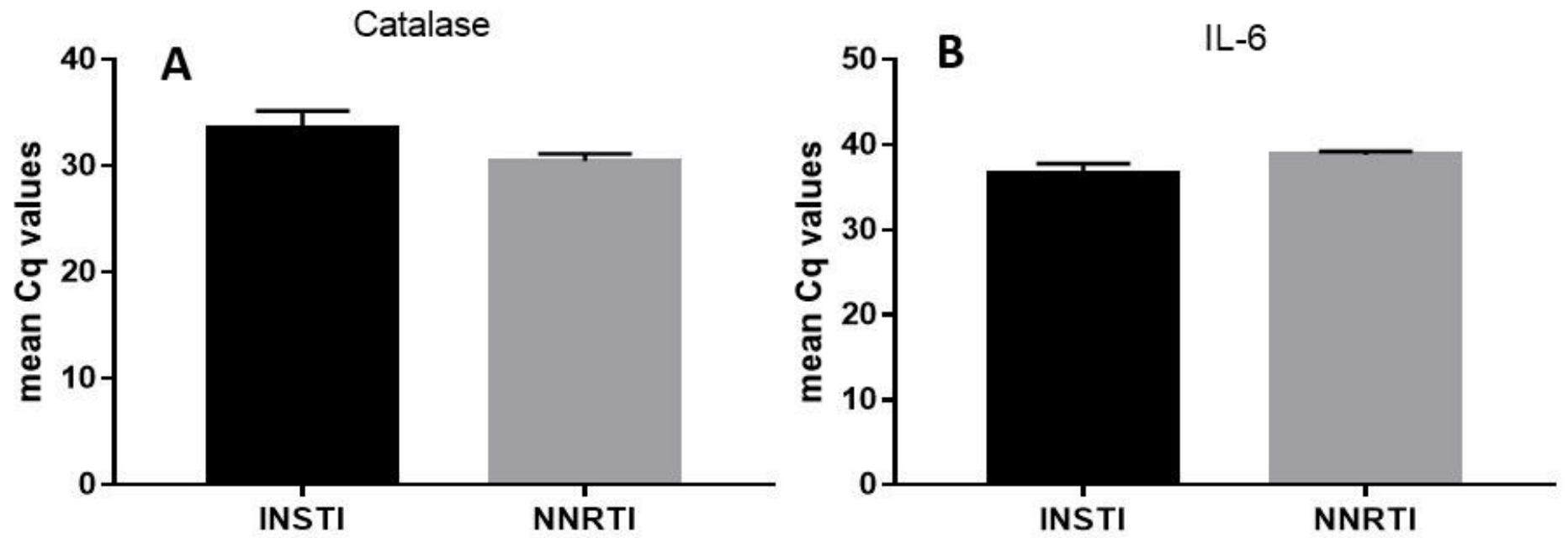

Figure 2

The effect of INSTI and NNRTI on CD34 +ve cell mRNA gene expressions: The important antioxidant gene Catalase (Fig.2A) expression increased $(p=0.13)$ in INSTI( $n=13)$ group as compared to NNRTI $(n=6)$ group. (Fig. 2A, $p=0.13)$. Concurrently, the inflammatory marker IL6 gene expression was lower $(p=0.06)$ in INSTI $(n=13)$ group as compared to NNRTI $(n=6)$ group, (Fig.2B, $p=0.06)$. However, the results did not reach statistical significance. 


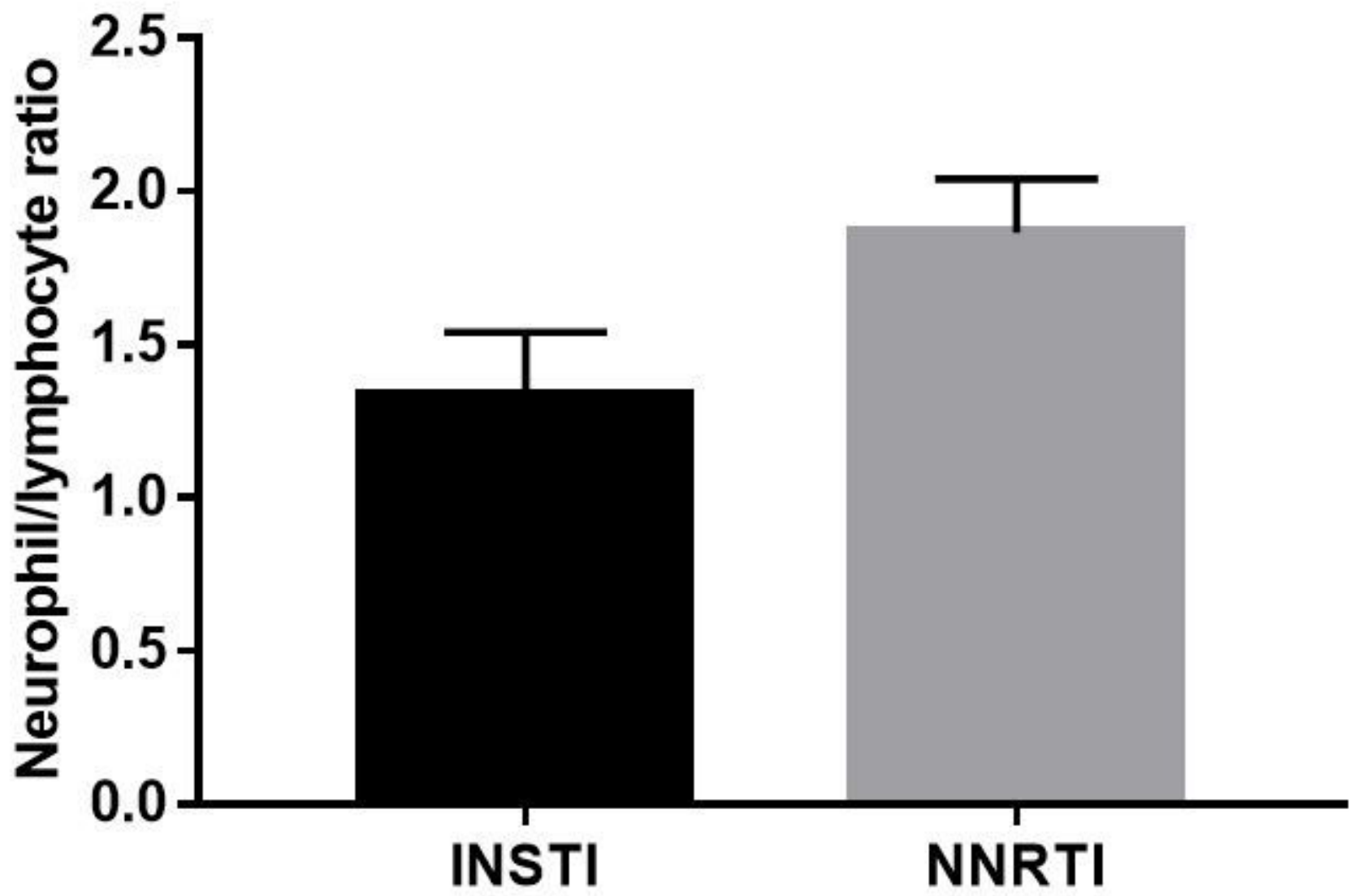

Figure 3

Neutrophil and lymphocyte ratio (NLR): A non-statistically significant decrease in NLR $(p=0.11)$ was observed in INSTI $(n=13)$ group as compared to NNRTI $(n=6)$ group. A decrease in NLR is associated with reduced inflammation. 


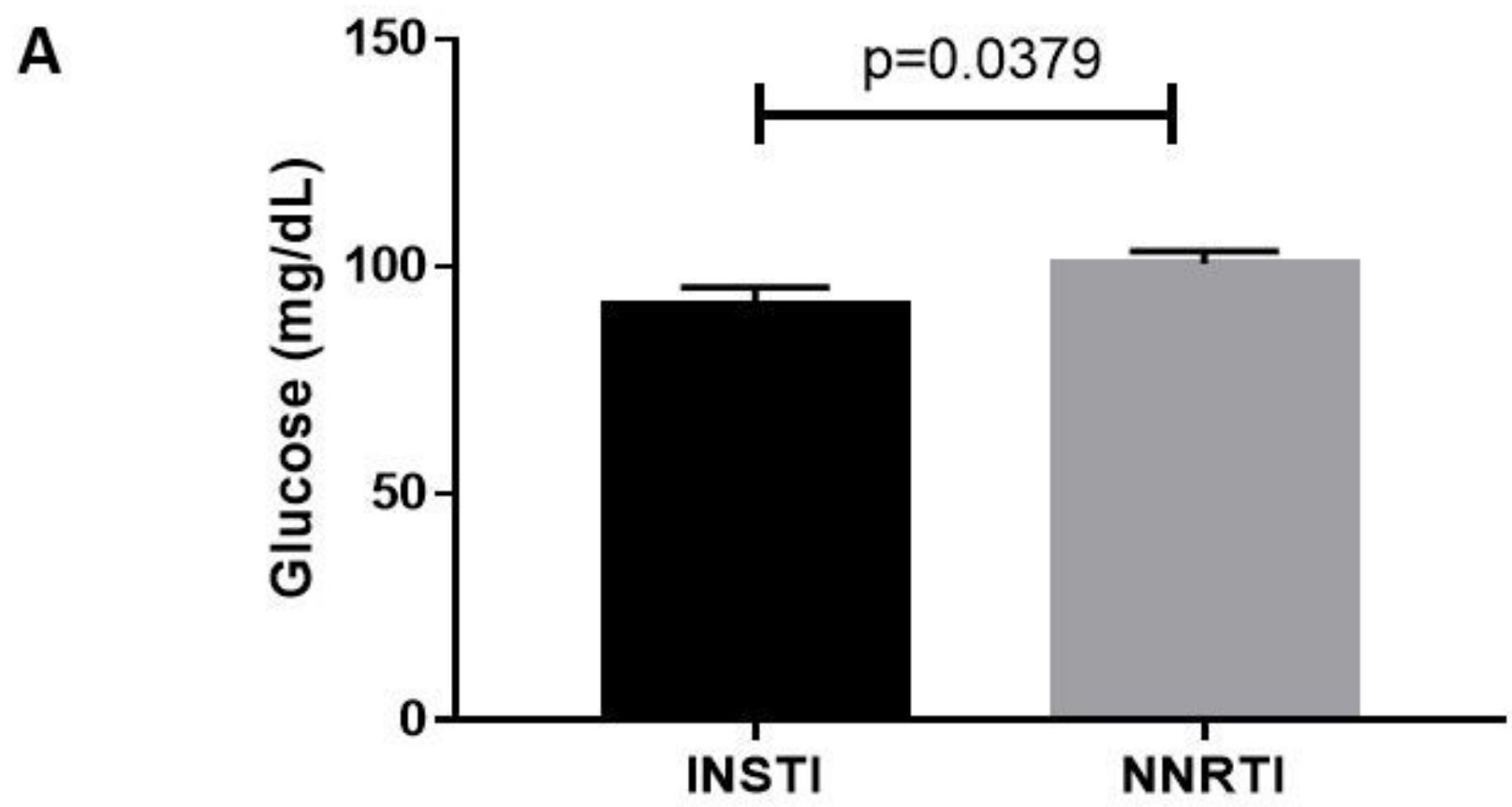

Figure 4

Effect on blood Glucose: Fasting Glucose levels(Fig.4A) were found to be lower in INSTI group, statistically significant at $p=0.037$. There was no difference in concomitant insulin values 


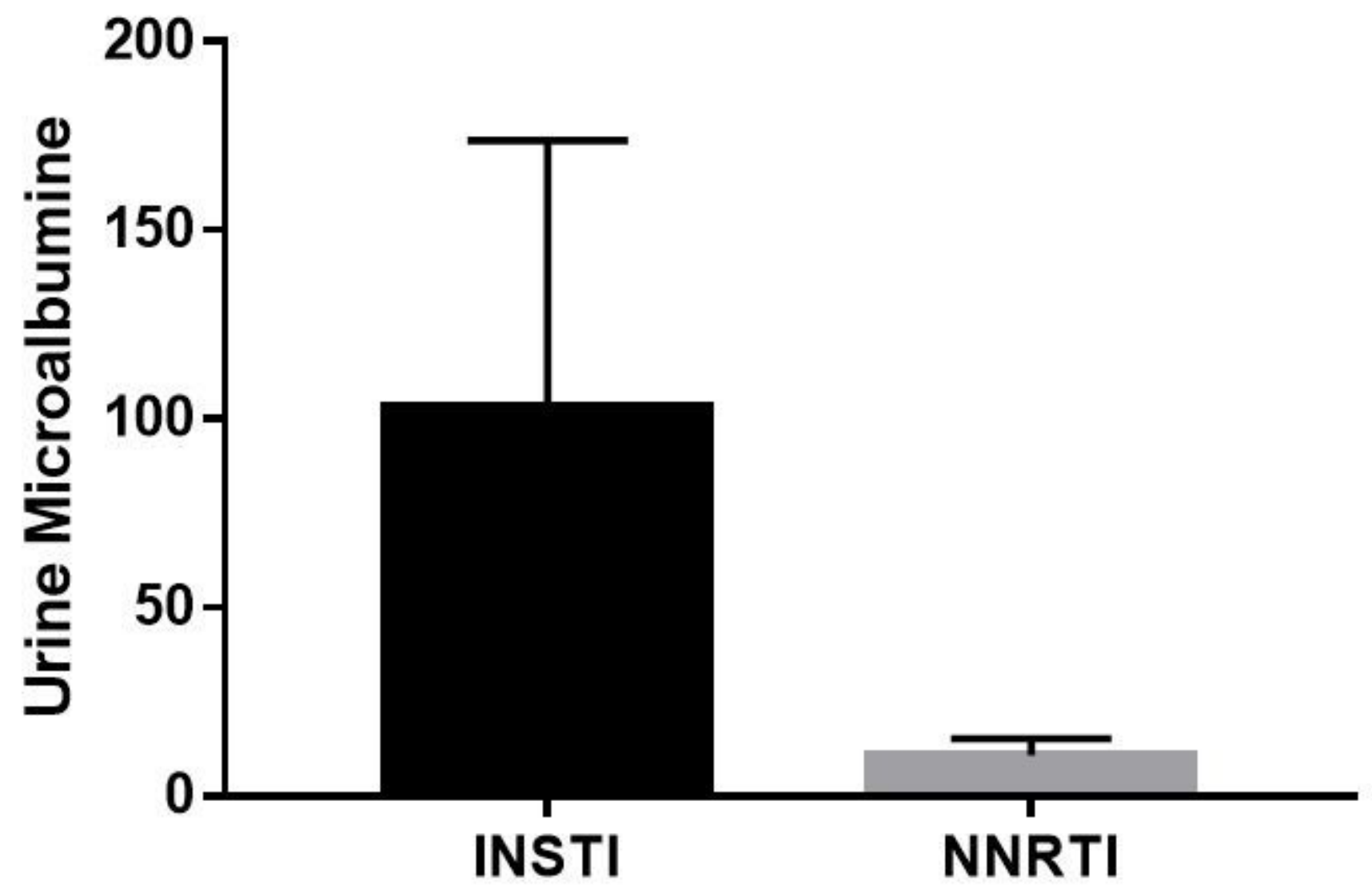

Figure 5

Urine Microalbumin: Interestingly, urine microalbumin levels were noted to be higher in $\operatorname{INSTI}(n=13)$ group as compared to NNRTI $(n=6)$ group. The result was close to statistical significance $(p=0.08)$. 


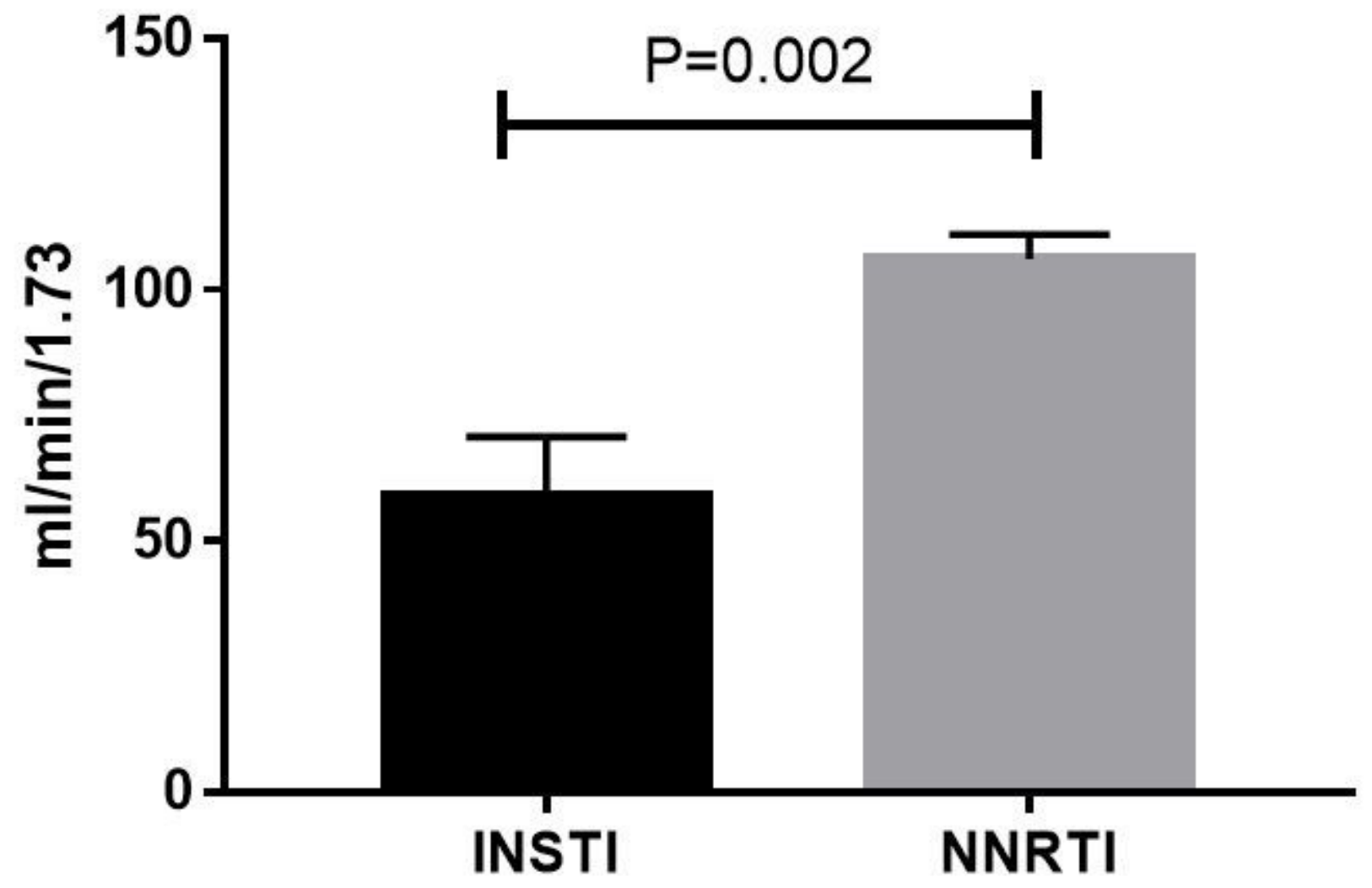

Figure 6

Glomerular Filtration Rate: As an index of kidney function, eGFR values showed normal levels in the NNRTI $(n=6)$ group compared to abnormally low levels in INSTI $(n=13)$ group, which maybe an indication of CKD. The results were statistically significant $(p=0.002)$.

\section{Supplementary Files}

This is a list of supplementary files associated with this preprint. Click to download.

- LetterHIVSCRTJan2022.docx 